\title{
Clinical and cognitive predictors of swallowing recovery in stroke
}

\author{
Mae Fern Schroeder, BA; ${ }^{1}$ Stephanie K. Daniels, PhD; ${ }^{1-2 *}$ Maryellen McClain, PhD; ${ }^{2}$ David M. Corey, PhD; ${ }^{2-3}$ \\ Anne L. Foundas, $\mathrm{MD}^{2,4}$ \\ ${ }^{1}$ Research Service, New Orleans Department of Veterans Affairs Medical Center (VAMC), New Orleans, LA; \\ ${ }^{2}$ Department of Psychiatry and Neurology, Tulane University Health Sciences Center, New Orleans, LA; ${ }^{3}$ Department \\ of Psychology, Tulane University, New Orleans, LA; ${ }^{4}$ Neurology Service, New Orleans VAMC, New Orleans, LA
}

\begin{abstract}
This retrospective study determined whether specific neurological features were associated with initial and final swallowing outcomes in acute stroke patients. A chart review of 65 acute stroke patients suggested that certain clinical and neurocognitive behaviors were associated with swallowing outcomes. Hemispatial neglect was significantly associated with initial nonoral dietary intake, whereas aphasia was not associated with swallowing outcome. Results from the initial clinical swallowing evaluations suggested that the presence of at least four of six clinical features (cough after swallow, voice change after swallow, abnormal volitional cough, abnormal gag reflex, dysphonia, and dysarthria) were associated with poor initial and final swallowing outcomes. Whether specific lesion location, size, or a combination of clinical neurological deficits are associated with poor initial and final swallowing outcomes is unclear. Prospective studies are warranted for further investigation of these relationships.
\end{abstract}

Key words: aphasia, aspiration, diet, dysphagia, hemispatial neglect, hemispheric damage, penetration, stroke, swallowing, videofluoroscopic swallow study.

\section{INTRODUCTION}

Dysphagia, an impairment of swallowing function, commonly occurs following acute stroke. Using various diagnostic methods and timing of evaluations poststroke, previous studies have shown that dysphagia affects up to 50 percent of acute stroke patients [1-2]. The degree of dysphagia persistence seems to depend on the method of evaluation. At 6 months poststroke, clinical swallowing evaluations show that 11 percent of patients have dysphagia [3], whereas videofluoroscopic swallow studies (VSSs) demonstrate that 50 percent have continued dysphagia [4]. The clinical swallowing evaluation is a noninstrumental assessment that is performed at bedside with various volumes and consistencies of material. Given that assessment of only swallowing will miss silent aspiration, i.e., no cough or voice change with aspiration [5], the clinical swallowing evaluation also assesses cranial nerves, oromotor strength and agility, cognition, speech, language, and voice. Depending on severity, patients with dysphagia may become malnourished or dehydrated and those who aspirate have an increased risk of developing pneumonia [6]. These factors, including changes in diet or feeding techniques, increase caregiver responsibility, affect the patient's quality of life, increase length of hospitalization [7], and increase the likelihood of discharge to a nursing-care facility rather than home [7-8].

\footnotetext{
Abbreviations: $\mathrm{ANOVA}=$ analysis of variance, $\mathrm{DV}=$ dependent variable, IV = independent variable, LHD = left-hemispheric damage, MRI = magnetic resonance imaging, $\mathrm{P}-\mathrm{A}=$ PenetrationAspiration, PET $=$ positron emission tomography, RHD = right-hemispheric damage, $\mathrm{SD}=$ standard deviation, $\mathrm{VAMC}=$ Department of Veterans Affairs medical center, VSS = videofluoroscopic swallow study.

*Address all correspondence to Stephanie K. Daniels, PhD; Research Service, New Orleans VAMC, 1601 Perdido Street, New Orleans, LA 70112-1261; 504-556-7225; fax: 504-556-7357.

Email:stephanie.daniels@med.va.gov

DOI: 10.1682/JRRD.2004.12.0154
} 
Although dysphagia is a major source of disability in stroke patients, its neurobiological basis is largely unknown. While unilateral stroke of either cerebral hemisphere is now clearly recognized to produce dysphagia, it is unclear whether one hemisphere is more dominant for swallowing and whether damage to a specific hemisphere affects swallowing recovery [9-11]. Findings from functional and anatomical imaging have been contradictory concerning swallowing lateralization. Magnetoencephalography has identified significantly greater activation of the left sensorimotor cortex during swallowing as compared with the right sensorimotor cortex, with the degree of lateralization dependent upon task complexity [12]. Studies that used transcranial magnetic stimulation and positron emission tomography (PET) suggest that swallowing is lateralized but representation of swallowing within subjects is asymmetrical [13-15]. That is, results suggest that swallowing is not lateralized to one specific hemisphere, but within individuals, one hemisphere tends to be more important than the other in mediating swallowing. Furthermore, lesion studies have demonstrated that swallowing behaviors may differ between left-hemispheric damage (LHD) and right-hemispheric damage (RHD) strokes. Specifically, LHD has been associated with oral-stage dysfunction, while RHD has been associated with pharyngeal-stage dysfunction and aspiration [10-11]. Conversely, other studies have not identified lateralization or hemispheric specialization of swallowing. Equal incidences of dysphagia, aspiration, and oral and pharyngeal dysmotility have been found in LHD and RHD stroke patients [9,16]. Moreover, bilateral symmetrical activation of the sensorimotor cortex has been identified in functional magnetic resonance imaging (MRI) and PET studies of healthy adults [17-18].

How RHD or LHD relates to swallowing recovery is currently unknown because only one study has used VSS to study swallowing acutely (1 to 4 days poststroke) and at 1 month poststroke. Smithard et al. found that patients with RHD were more likely to have persistent dysphagia than patients with LHD [19]. When evaluated acutely, four patients with RHD and nine patients with LHD had aspirated $(N=87)$; however, at 1 month, seven patients with RHD and no patients with LHD had aspirated. One should note that the neuroimaging scan was not completed at 1 month poststroke. While the authors do not account for the increased incidence of aspiration in RHD patients at 1 month, various factors such as worsened swallowing or an undetected stroke may have occurred in these patients.

In addition to the location of the lesion, lateralized neurocognitive deficits, such as aphasia and hemispatial neglect, may be related to acute and protracted dysphagia. Considerable empirical evidence supports the presence of aphasia more commonly in LHD than RHD patients and the presence of hemispatial neglect more commonly in RHD than LHD patients [20]; therefore, study of these two deficits may prove important in determining which patients are at risk for acute and protracted dysphagia. However, the association of lateralized neurocognitive deficits with dysphagia has been minimally studied, with no known study having used VSS to confirm dysphagia. Barer found that speech intelligibility, response to commands, visual-field deficits, and sensory inattention were strongly correlated with clinically determined acute dysphagia [21]. Unfortunately, the relationship of these neurological variables with dysphagia was not assessed at the 6-month follow-up. Thus, whether neurocognitive behaviors are associated with acute or protracted RHD or LHD and dysphagia remains unclear.

Although instrumental studies remain the gold standard [22], clinical swallowing evaluations have been validated for determining aspiration risk in patients and for identifying which stroke patients warrant an instrumental examination. Daniels et al. developed a clinical swallowing evaluation with the following six clinical features of aspiration risk in acute stroke patients: dysphonia, dysarthria, abnormal volitional cough, abnormal gag reflex, cough after swallow, and voice change after swallow [1,23-24]. Numerous studies have found that some or all of these features are related to risk of aspiration [25-28]. These features are independent in that a person may demonstrate one feature in isolation without the presence of an additional feature [1,23-24]. Even though these features may appear to overlap, for example, dysphonia may be part of a dysarthria, each offers unique information and is important in the clinical evaluation of swallowing. Furthermore, the presence of any two of these six clinical predictors is strongly associated with the identification of laryngeal penetration with stasis to silent aspiration on VSS [23-24]. While clinical screenings have demonstrated good sensitivity and specificity in identifying aspiration risk in acute stroke patients, whether the number of specific clinic predictors is associated with identification of protracted dysphagia is unclear.

Our major aim was to determine whether specific neurological predictors were associated with dysphagia and recovery in acute stroke patients. We used three outcomes to define dysphagia: Penetration-Aspiration (P-A) Scale score [29], dysphagia severity, and diet, all of which are detailed in the "Methods" section. Based on Barer's findings [21], we hypothesized that hemispatial neglect 
(right-hemisphere lateralized behavior) would be associated with acute and chronic dysphagia as identified by validated noninstrumental techniques and clinical assessment. We predicted that aphasia (left-hemisphere lateralized behavior) would not be associated with acute or persistent dysphagia because Barer did not look at aphasia as a single entity but rather grouped it with dysphonia and dysarthria [21]. Based on our prior research that showed that dysphonia and dysarthria were associated with aspiration risk in acute stroke patients [1], we posited that these two features, not aphasia, provided the association with dysphagia in Barer's study [21]. Given that identification of two of six clinical features is associated with aspiration risk in acute stroke patients [23], we predicted that identification of an increased number of features on the initial clinical swallowing evaluation would be associated with poor initial and final swallowing outcomes. Based on previous research $[9,16]$, we predicted that initial and final swallowing outcomes would not differ in patients with RHD versus LHD but patients with posterior circulation territory lesions would have worse swallowing outcomes [22].

\section{METHODS}

\section{Subjects}

We retrospectively reviewed the medical records of 65 acute stroke patients who had been consecutively referred to and undergone a clinical swallowing evaluation by the Speech Pathology Service at the New Orleans Department of Veterans Affairs Medical Center (VAMC) between January 2001 and December 2002. Patients with a history of head and neck structural damage, neurological disease other than stroke, and a current history of dysphagia were excluded from the study. Patients who had demonstrated dysphagia after a previous stroke but had documented resolution of the dysphagia were included in the study. Patients ranged in age from 42 to 101 years with a mean age of 67 (standard deviation $[\mathrm{SD}]=13$ ). The study was approved by the Tulane University Health Sciences Center Institutional Review Board and the New Orleans VAMC.

The computed tomography or MRI scan of each patient was reviewed. A total of 35 patients (54\%) had had a single unilateral stroke; of these 35 patients, 18 (51\%) had RHD and 17 (49\%) had LHD. Thirty patients ( $46 \%$ of the sample) had a history of prior stroke. Lesion location of the new stroke was classified in all 65 patients and included RHD ( $n=30,46 \%)$, LHD ( $n=29,45 \%)$, and posterior circulation territory ( $n=6,9 \%)$, which included the brain stem, pons, and cerebellum.

Medical records were reviewed for the presence or absence of hemispatial neglect and aphasia. Testing for these deficits was completed by the admitting neurologist and/or during the speech pathology evaluation. Hearing was not objectively evaluated, but no patient demonstrated obvious deficits that affected testing. Tasks for evaluation of hemispatial neglect included line bisection, cancellation, and extinction. Based on errors in these tasks, such as failure to cross out stimuli on one side in the cancellation task or to respond to one side with double simultaneous stimulation, we classified patients with neglect if they demonstrated an acute onset of decreased attention to one visual field in two of the three tasks. Patient comprehension, fluency, naming, and repetition were used for determining the presence of aphasia. Fluency was defined as easy, plentiful verbalization with good articulatory agility, normal phrase length (five to eight words each phrase), variety of grammatical constructions, normal prosody, and good naming without paraphasic errors [30]. A patient was defined as aphasic if they demonstrated an acute onset of deficits in at least one of the four language areas (comprehension, fluency, naming, and repetition). Since patients with aphasia may or may not also present with dysarthria, we used motorspeech impairment tasks that assess articulation, rate, intensity, and resonance to determine the presence of dysarthria and language tasks that assess grammatical form, phrase length, content, melodic line, articulatory agility, and word finding to determine nonfluent aphasia.

Hemispatial neglect was identified in 15 (23\%) and aphasia was identified in 16 (25\%) of the 65 patients. Of the 15 patients with hemispatial neglect, 11 (73\%) had RHD and 4 (27\%) had LHD. Of the 16 patients with aphasia, 14 (88\%) had LHD, 1 (6\%) had RHD, and 1 (6\%) had an infarct that involved the posterior circulation territory. In all patients except one, the diagnosis of hemispatial neglect or aphasia was new and associated with the acute stroke. One patient who had had a second left-hemispheric stroke had worsened aphasia from his preadmission baseline.

\section{Procedures}

All patients underwent a clinical swallowing evaluation within 3 days of admission. As part of this evaluation, dysphonia, dysarthria, abnormal gag reflex, abnormal volitional cough, cough after swallow, and voice change after swallow (Table 1) were assessed and 
JRRD, Volume 43, Number 3, 2006

Table 1.

Clinical predictors of aspiration risk.

\begin{tabular}{lr}
\hline \multicolumn{1}{c}{ Predictor } & \multicolumn{1}{c}{ Operational Definition } \\
\hline $\begin{array}{l}\text { Dysphonia } \\
\text { Dysarthria }\end{array}$ & $\begin{array}{l}\text { Voice disturbance in parameters of vocal quality, pitch, or intensity. } \\
\text { Speech disorder resulting from disturbances in muscular control that affect respiration, articula- } \\
\text { tion, phonation, resonance, or prosody. } \\
\text { Abnormal Gag Reflex }\end{array}$ \\
$\begin{array}{l}\text { Absent or weakened velar or pharyngeal wall contraction, unilaterally or bilaterally, in response } \\
\text { to tactile stimulation of posterior pharyngeal wall. }\end{array}$ \\
$\begin{array}{l}\text { Abnormal Volitional Cough } \\
\text { Cough After Swallow }\end{array}$ & Weak, verbalized, or absent response upon command to cough. \\
& Cough immediately after or within 1 min of ingestion of calibrated volumes of water (5, 10, and \\
Voice Change After Swallow & Alteration in vocal quality after ingestion of calibrated volumes of water. \\
\hline \hline
\end{tabular}

scored on a binary present/absent basis. The total number of clinical features present for each patient was identified.

Consistent with the clinical pathway used at the New Orleans VAMC, patients who demonstrated two or more features of aspiration risk on the clinical swallowing evaluation were further evaluated with a VSS $(n=36)$. Patients who demonstrated less than two clinical features were not evaluated with a VSS $(n=29)$. The VSS was completed with a standard protocol detailed previously [1,23]. Briefly, a video recording of the oral cavity and pharynx was obtained in the lateral plane as patients swallowed in duplicate 3, 5, 10, or $20 \mathrm{~mL}$ of liquid barium and $5 \mathrm{~mL}$ of barium paste. In addition, patients masticated and swallowed half of a barium-coated cookie and sequentially swallowed $100 \mathrm{~mL}$ of liquid barium. All examinations were initiated with the $3 \mathrm{~mL}$ volume and advanced accordingly unless the patient exhibited significant aspiration that could not be eliminated with therapeutic intervention. In these cases, the study was terminated. The initial VSS was completed within the first 5 days of admission. Follow-up VSSs were completed as clinically warranted. A recording of the VSS was used for scoring airway invasion with the P-A Scale, an ordinal scale that measures depth of airway invasion, clearance, and response to airway invasion (Table 2) [29]. The highest P-A Scale score was recorded and then classified as normal-mild (score of 1-2), moderate (score of 3-5), and severe (score of 6-8). Inter- and intrarater reliabilities for P-A Scale score were completed in 10 randomly selected patients (intraclass correlation coefficients $=0.994$ and 0.947 , respectively). In addition, the P-A Scale was used for determination of dysphagia severity based on the number of aspiration occurrences and the consistencies aspirated (Table 3). Speech pathology reports from the initial and final VSSs of the patient's hospitalization determined the outcome measures of initial
Table 2.

Grouping and description of Penetration-Aspiration Scale scores.

\begin{tabular}{|c|c|c|}
\hline Score & Classification & Description \\
\hline 1 & Normal & No airway invasion. \\
\hline 2 & Mild & Bolus enters airway with clearing \\
\hline 3 & Moderate & $\begin{array}{l}\text { Bolus enters airway without } \\
\text { clearing. }\end{array}$ \\
\hline 4 & Moderate & $\begin{array}{l}\text { Bolus contacts vocal cords with } \\
\text { airway clearing. }\end{array}$ \\
\hline 5 & Moderate & $\begin{array}{l}\text { Bolus contacts vocal cords with- } \\
\text { out airway clearing. }\end{array}$ \\
\hline 6 & Severe & $\begin{array}{l}\text { Bolus enters trachea and is cleared } \\
\text { into larynx or out of airway. }\end{array}$ \\
\hline 7 & Severe & $\begin{array}{l}\text { Bolus enters trachea and is not } \\
\text { cleared despite patient attempts. }\end{array}$ \\
\hline 8 & Severe & $\begin{array}{l}\text { Bolus enters trachea and patient } \\
\text { does not attempt to clear. }\end{array}$ \\
\hline
\end{tabular}

Table 3.

Classification of dysphagia severity.

\begin{tabular}{|c|c|}
\hline Classification & Description \\
\hline Normal-Mild & $\begin{array}{l}\text { Range from no laryngeal penetration to } \\
\text { evidence of laryngeal penetration. }\end{array}$ \\
\hline Moderate & $\begin{array}{l}\text { Two or less aspiration episodes of one } \\
\text { consistency. }\end{array}$ \\
\hline Severe & $\begin{array}{l}\text { More than two aspiration episodes of one } \\
\text { consistency or aspiration of more than } \\
\text { one consistency. }\end{array}$ \\
\hline
\end{tabular}

and final P-A Scale scores and dysphagia severity for each patient.

Patient records were also reviewed for dietary status following the initial swallowing evaluation and at discharge. Diet recommendations were based on the results of the clinical swallowing evaluation and the VSS and included regular, mechanical soft, pureed, thickened liquids, 
or nonoral intake. For this study, diet was stratified into two groups following the initial swallowing assessment: oral and nonoral. The diet at discharge, as well as any patient death prior to discharge, was also recorded and stratified into two groups: oral and nonoral/deceased.

\section{Statistical Analyses}

We used chi-square $\left(\chi^{2}\right)$ tests of independence to test the relationships between all dependent variables (DVs) and the independent variables (IVs) of aphasia, hemispatial neglect, and lesion location. The DVs measured were-

1. Initial diet (oral, nonoral; determined after the clinical and/or VSS evaluations).

2. Final diet (oral, nonoral/death; determined at discharge).

3. Initial P-A Scale score (normal-mild, moderate, severe).

4. Final P-A Scale score (normal-mild, moderate, severe).

5. Initial dysphagia severity score (normal-mild, moderate, severe).

6. Final dysphagia severity score (normal-mild, moderate, severe).

Initial and final dysphagia severity scores were based on the P-A Scale scores and then categorized as normalmild, moderate, and severe dysphagia. Final P-A Scale and dysphagia severity scores were obtained from the last VSS during hospitalization. An additional IV, number of clinical features present on the clinical swallowing examination, was analyzed by analysis of variance (ANOVA) with the same six DVs as the chi-square analyses. Note that testing of these relationships by ANOVA required prediction of number of features from the DVs, rather than conventional prediction of the DVs by the IVs. Thus, each ANOVA evaluated the amount of variance in number of clinical features that could be explained by a DV.

Because associations with six DVs were tested for each IV, Bonferroni corrections were applied as follows:

- Tests of IVs that measure lateralized behaviors (aphasia and neglect) were taken as a unit that included 12 significance tests and yielded corrected $\alpha=0.0042$ (0.05/12).

- Number of clinical features and lesion location were corrected independently with six significance tests each and yielded corrected $\alpha=0.0083(0.05 / 6)$.

\section{RESULTS}

\section{Swallowing Outcomes}

Based on results of the clinical swallowing evaluation and/or initial VSS, 41 (63\%) of the 65 patients were recommended for oral intake and 24 (37\%) for nonoral intake. By discharge (which ranged from 3 days to 4 months after admission), 49 (75\%) of the 65 patients were receiving oral intake, 6 (9\%) were receiving nonoral intake, and 10 (16\%) had died. Six of the patients who died had been diagnosed with aspiration pneumonia, which may have contributed to their deaths.

Thirty-six patients underwent an initial VSS following the clinical swallowing evaluation. Of these, 9 (25\%) presented with normal-to-mild initial P-A Scale scores (score of 1-2), 9 (25\%) presented with a moderate P-A Scale scores (score of 3-5), and 18 (50\%) presented with severe P-A Scale scores (score of 6-8) that indicated at least one aspiration episode. Furthermore, the initial dysphagia severity score, as determined by the number of aspiration episodes and consistencies aspirated, was classified as normal-mild in 17 patients (47\%), moderate in 5 (14\%), and severe in 14 (39\%). Ten patients underwent at least one repeat VSS. Of these 10 patients, 4 (40\%) improved to within the normal-mild range on the final P-A Scale score and 1 (10\%) improved to within the moderate range; however, 5 (50\%) remained in the severe range. Of these 10 patients, the final dysphagia severity score was normal-mild in 5 (50\%), moderate in 3 (30\%), and severe in $2(20 \%)$.

\section{Lateralized Neurocognitive Behaviors}

Data are summarized in Table 4. Hemispatial neglect was significantly associated with initial $\operatorname{diet}\left(\chi^{2}{ }_{1}=11.10\right.$, $p<0.001)$ but not with final diet $\left(\chi^{2}{ }_{1}=2.49, p=0.12\right)$, initial P-A Scale score $\left(\chi_{2}^{2}=1.37, p=0.5\right)$, final P-A Scale score $\left(\chi_{2}^{2}=1.88, p=0.39\right)$, initial dysphagia severity score $\left(\chi^{2}{ }_{2}=4.54, p=0.1\right)$, or final dysphagia severity score $\left(\chi_{2}^{2}=0.14, p=0.93\right)$. That is, the presence of hemispatial neglect was associated with nonoral intake at the initial swallowing evaluation but not with poor final swallowing outcome at discharge, P-A Scale scores, or dysphagia severity scores.

Aphasia was not significantly associated with initial $\operatorname{diet}\left(\chi^{2}{ }_{1}=0.43, p=0.52\right)$, final diet $\left(\chi^{2}{ }_{1}=7.37, p=\right.$ $0.007)$, initial P-A Scale score $\left(\chi_{2}^{2}=3.07, p=0.22\right)$, or initial dysphagia severity score $\left(\chi^{2} 2=8.24, p=0.02\right)$. Analyses for association of aphasia with final P-A Scale 
Table 4.

Number (percent) of participants assigned to oral versus nonoral diets at initial and final evaluations.

\begin{tabular}{lcccccc}
\hline \multicolumn{1}{c}{ Patient } & \multirow{2}{*}{ Group } & $\boldsymbol{n}$ & \multicolumn{2}{c}{ Initial Diet } & & \multicolumn{2}{c}{ Final Diet } \\
\cline { 3 - 4 } \cline { 5 - 6 } & & Oral & Nonoral & & Oral & Nonoral \\
\hline $\begin{array}{l}\text { Hemispatial } \\
\text { Neglect }\end{array}$ & 15 & $4(27)$ & $11(73)$ & & $9(60)$ & $6(40)$ \\
Aphasia & 16 & $9(56)$ & $7(44)$ & & $8(50)$ & $8(50)$ \\
\hline \hline
\end{tabular}

score or dysphagia severity score could not be computed because the 10 patients with final test scores were categorized as nonaphasic.

\section{Clinical Swallowing Features}

Patients who initially received oral intake demonstrated significantly fewer clinical features (mean $\pm \mathrm{SD}=$ $2.20 \pm 1.47)$ than those who initially received nonoral intake (mean $\pm \mathrm{SD}=3.89 \pm 1.02, F_{1,57}=19.63, p<$ 0.001). Similarly, the number of clinical features present in the final examination was significantly fewer for those who received oral intake (mean $\pm \mathrm{SD}=2.35 \pm 1.39$ ) versus nonoral intake (mean $\pm \mathrm{SD}=4.50 \pm 0.97, F_{1,57}=$ $21.60, p<0.001$ ). These significant results are summarized in Table 5. The number of clinical features was not associated with initial P-A Scale score $\left(F_{2,33}=1.24, p=\right.$ $0.3)$, final P-A Scale score $\left(F_{2,7}=0.49, p=0.63\right)$, initial dysphagia severity score $\left(F_{2,33}=3.56, p=0.04\right)$, or final dysphagia severity score $\left(F_{2,7}=0.545, p=0.6\right)$.

\section{Lesion Location}

Lesion location (LHD, RHD, or posterior circulation territory) was not significantly associated with initial P-A Scale score $\left(\chi^{2}{ }_{4}=1.50, p=0.83\right)$, final P-A Scale score $\left(\chi_{2}^{2}=1.43, p=0.49\right)$, initial dysphagia severity score $\left(\chi^{2}{ }_{4}=5.44, p=0.25\right)$, final dysphagia severity score $\left(\chi_{2}^{2}=1.11, p=0.58\right)$, initial diet $\left(\chi_{2}^{2}=0.23, p=0.89\right)$, or final diet $\left(\chi_{2}^{2}=0.73, p=0.8\right)$.

\section{DISCUSSION}

Given that dysphagia can affect quality of life and prolong hospitalization, identification of factors that may predict which stroke patients are at risk for acute and persistent dysphagia is important. A better understanding of factors that may predict and affect recovery will expedite evaluation and treatment and reduce complications. The results of this study suggest that specific lateralized
Table 5.

Number of clinical swallowing features at clinical evaluation (mean \pm standard deviation). Scores varied significantly with diet type at initial and final evaluations (both $p<0.001$ ).

\begin{tabular}{ccc}
\hline \multirow{2}{*}{ Evaluation } & \multicolumn{2}{c}{ Diet Type } \\
\cline { 2 - 3 } & Oral & Nonoral \\
\hline Initial & $2.20 \pm 1.47$ & $3.89 \pm 1.02$ \\
Final & $2.35 \pm 1.39$ & $4.50 \pm 0.97$ \\
\hline \hline
\end{tabular}

neurocognitive deficits and the number of clinical features identified on a clinical swallowing evaluation are associated with swallowing outcomes. The presence of hemispatial neglect was significantly associated with initial nonoral intake. Moreover, the identification of at least four clinical features on initial dysphagia screening was associated with poor initial and final diet outcomes.

To our knowledge, no research has specifically examined the relationship between lateralized neurocognitive deficits (e.g., hemispatial neglect and aphasia) and acute and chronic dysphagia. In a study of stroke and persistent dysphagia, Barer evaluated visual extinction and speechlanguage skills among a host of other features [21]; however, this study did not focus on lateralized deficits and objective measures (i.e., validated clinical evaluation and VSS) were not used for swallowing evaluation. Visual extinction, a symptom found in some patients with hemispatial neglect, was associated with acute dysphagia. Our results demonstrated that hemispatial neglect, as measured by line bisection, cancellation, and extinction, was associated with objectively identified acute dysphagia and yielded increased nonoral intake. Our study differed from Barer's in that we used a more rigorous clinical assessment of neglect and confirmed dysphagia with VSS. Unlike Barer, we did not find an association between aphasia and dysphagia. Our results indicated that aphasia was relatively equally distributed among patients who received oral versus nonoral intake. One should note that Barer used aphasia, dysphonia, and dysarthria to classify dysphagia. Thus, the exact relationship between aphasia and dysphagia remains unclear because motorspeech and voice deficits were also included in the rating and because the correlation of dysarthria and dysphonia with aspiration risk is well established [1,23-28]. These results do not suggest that clinicians use the presence or absence of hemispatial neglect or aphasia to determine which patients warrant a VSS. Rather, the results suggest that patients with hemispatial neglect may be more likely than patients with aphasia to require initial nonoral 
intake; however, patients should have an objective swallowing evaluation prior to their assignment to a nonoral diet. One should note that the present study included Bonferroni correction, which produces very conservative significance tests. Therefore, the relationship between aphasia and diet outcomes may exist but possibly was not detected here because of a lack of statistical power. Our interpretation that this relationship is absent is therefore offered tentatively and will require substantiation by a future study with greater statistical power.

While we used a hemispheric approach to define cognition, neurocognitive functions may be better conceptualized from a systems approach. Although cognitive deficits are frequently associated with specific hemispheres, they can occur with deficits in the neural systems that underlie these cognitive functions, which are not strictly hemispheric in nature. Our findings support a systems approach because neglect and aphasia were identified, albeit very infrequently, in atypical hemisphere lesions.

Because this was a retrospective study and neurocognitive deficits were not reassessed, any relationship between recovery or decline of neurocognitive function and long-term swallowing outcomes cannot be determined. Furthermore, we did not examine additional neurocognitive behaviors such as apraxia and visuospatial functions. Ideomotor, limb, or buccofacial apraxia, as well as apraxia of speech, may be associated with acute and protracted dysphagia and should be studied. Future studies should prospectively study neurocognitive function in acute and chronic stroke patients.

Clinical swallowing evaluations, including oromotor and bedside swallowing tests, have been used for determination of the features associated with aspiration risk in acute stroke patients [1,23-28]. These studies did not follow patients longitudinally to determine whether specific deficits or combinations of deficits predicted persistent dysphagia. Previous research has shown that the presence of at least two of six clinical features is associated with an increased risk of aspiration during a VSS [23]. Our current study expands these results and suggests that the presence of any four clinical features may indicate poor acute and chronic outcomes. Patients who received oral intake averaged two clinical features, whereas patients with poor initial and final outcomes (nonoral intake/ death) averaged four clinical features. No significant relationships were observed between the number of clinical features and P-A Scale score or dysphagia severity. This may be, in part, because only airway invasion was considered and thus yielded highly specific outcomes. Stroke patients may demonstrate dysphagia and aspiration but not the poor swallowing outcomes of nonoral intake or death. Airway invasion may be limited by the amount or number of occurrences or alleviated with a compensatory strategy, such as a chin-tuck posture; thus, a person with aspiration may still receive oral intake. On the other hand, factors such as pharyngeal residue, cognitive status, and response to compensatory strategies influence decisions regarding diet and thus make diet a broader outcome. All stroke patients with dysphagia and risk of aspiration warrant a VSS to delineate the underlying physiological etiology of the dysphagia, determine the effects of compensatory strategies, and identify appropriate evidence-based treatments; thus, continued use of the presence of two or more clinical features as a guideline is strongly advocated. However, if a patient demonstrates four or more clinical features, poor diet outcome may be anticipated. One should note that clinical swallowing evaluations were not completed at follow-up, so any improvement or decline in clinical features is unknown. Thus, how changes in the presence or frequency of clinical features relate to swallowing outcomes is unclear. As we have previously noted, the absence of significant relationships may also result from inadequate statistical power. Relationships between number of clinical features and P-A Scale score (initial or final) or number of clinical features and dysphagia severity may exist but were not detected because of the conservative nature of Bonferroni-corrected significance tests. While we suggest that these relationships be interpreted as absent, substantiation of these results by further research is warranted.

Lesion location was not significantly associated with swallowing outcomes. This finding was consistent with results from prior studies [16], and our lesion-mapping in acute stroke patients has not indicated differences in swallowing dysfunction (oral dysmotility, pharyngeal dysfunction, aspiration) in patients with RHD versus LHD [9]. One may question why hemispatial neglect, generally a result of RHD, was related to poor outcomes but RHD was not. It is important to emphasize that RHD does not always result in hemispatial neglect. While 30 of the 65 patients had RHD, only 11 of these RHD patients demonstrated hemispatial neglect. As we have previously noted, a systems approach rather than a hemispheric approach may be preferable for conceptualizing "lateralized" behavior. Posterior circulation territory infarcts were also not associated with poor initial or final swallowing outcomes in our study. This finding is incongruent with prior research that identified severe and 
protracted dysphagia in patients with brain stem lesions, especially lesions that involved the dorsal and ventral regions of the medulla [22,31]. In our study, relatively few patients had posterior circulation territory lesions $(n=6)$ and we did not distinguish between medullary and midbrain lesions, which may have affected results. One should note that we neither identified precise lesion location or size in these patients nor studied the effects of multiple unilateral or bilateral strokes on swallowing. Thus, whether lesion size, lesion location, or a combination of these factors in conjunction with clinical and neurocognitive deficits are related to poor swallowing outcomes is unclear. Furthermore, we did not track which patients did and did not receive swallowing therapy. While the impact of therapy would not have influenced our initial findings, treatment may have affected our final findings and therefore should be studied in the future. Further prospective studies are warranted for investigation of these relationships.

\section{CONCLUSIONS}

Results from this retrospective study indicate that hemispatial neglect and the presence of at least four clinical features of dysphagia are associated with poor swallowing outcomes. Aphasia was not associated with swallowing outcomes. Neurocognitive deficits and clinical swallowing features were not reassessed, thus recovery or decline of function cannot be related to long-term outcomes in this study. Additional neurocognitive behaviors (e.g., limb apraxia) may also be associated with acute and protracted dysphagia and appropriate assessments of such should be incorporated into future studies. Lesion location was not significantly associated with any swallowing outcome; however, precise location and lesion size were not identified. Thus, whether neurocognitive deficits, lesion size, location, or a combination of these factors are related to poor swallowing outcomes is unclear.

\section{ACKNOWLEDGMENTS}

This material was based on work supported by a Department of Veterans Affairs Rehabilitation Research and Development Career Development Award to Dr. Daniels, grant B3019V.

The authors have declared that no conflicting interests exist.

\section{REFERENCES}

1. Daniels SK, Brailey K, Preistly DH, Herrington LR, Weisberg LA, Foundas AL. Aspiration in patients with acute stroke. Arch Phys Med Rehabil. 1998;79(1):14-19. [PMID: 9440410]

2. Gordon C, Hewer RL, Wade DT. Dysphagia in acute stroke. Br Med J (Clin Res Ed). 1987;295(6595):411-14. [PMID: 3115478]

3. Smithard DG, O’Neill PA, England RE, Park CL, Wyatt R, Martin DF, Morris J. The natural history of dysphagia following stroke. Dysphagia. 1997;12(4):188-93. [PMID: 9294937]

4. Mann G, Hankey GJ, Cameron D. Swallowing function after stroke: prognosis and prognostic factors at 6 months. Stroke. 1999;30(4):744-48. [PMID: 10187872]

5. Splaingard ML, Hutchins B, Sulton LD, Chaudhuri G. Aspiration in rehabilitation patients: videofluoroscopy vs bedside clinical assessment. Arch Phys Med Rehabil. 1988; 69(8):637-40. [PMID: 3408337]

6. Cesar L, Gonzalez C, Calia FM. Bacteriologic flora of aspiration-induced pulmonary infections. Arch Intern Med. 1997;135(5):711-14. [PMID: 28705$]$

7. Smithard DG, O’Neill PA, Park CL, Morris J. Complications and outcome after acute stroke. Does dysphagia matter? Stroke. 1996;27(7):1200-1204. [PMID: 8685928]

8. Odderson IR, Keaton JC, McKenna BS. Swallow management in patients on an acute stroke pathway: quality is cost effective. Arch Phys Med Rehabil. 1995;76(12):1130-33. [PMID: 8540789]

9. Daniels SK, Foundas AL. Lesion location in acute stroke patients with risk of aspiration. J Neuroimaging. 1999;9(2): 91-98. [PMID: 10208106]

10. Robbins JA, Levine RL. Swallowing after unilateral stroke of the cerebral cortex: preliminary experience. Dysphagia. 1988;3(1):11-17. [PMID: 3248391]

11. Robbins JA, Levine RL, Maser A, Rosenbek JC, Kempster GB. Swallowing after unilateral stroke of the cerebral cortex. Arch Phys Med Rehabil. 1993;74(12):1295-1300. [PMID: 8259895]

12. Dziewas R, Soros P, Ishii R, Chau W, Henningsen H, Ringelstein EB, Knecht S, Pantev C. Neuroimaging evidence for cortical involvement in the preparation and in the act of swallowing. Neuroimage. 2003;20(1):135-44. [PMID: 14527576]

13. Hamdy S, Aziz Q, Rothwell JC, Singh KD, Barlow J, Hughes DG, Tallis RC, Thompson DG. The cortical topography of human swallowing musculature in health and disease. Nat Med. 1996;2(11):1227-24. [PMID: 8898748$]$

14. Hamdy S, Aziz Q, Rothwell JC, Crone R, Hughes DG, Tallis RC, Thompson DG. Explaining oropharyngeal dysphagia after unilateral hemispheric stroke. Lancet. 1997;350(9079): 686-92. [PMID: 9291902] 
15. Hamdy S, Rothwell JC, Brooks DJ, Bailey D, Aziz Q, Thompson DG. Identification of the cerebral loci processing human swallowing with $\mathrm{H}_{2}{ }^{15} \mathrm{O}$ PET activation. J Neurophysiol. 1999;81(4):1917-26. [PMID: 10200226$]$

16. Alberts MJ, Horner J, Gray L, Brazer SR. Aspiration after stroke: lesion analysis by brain MRI. Dysphagia. 1992; 7(3):170-73. [PMID: 1499361]

17. Martin RE, Goodyear BG, Gati JS, Menon RS. Cerebral cortical representation of automatic and volitional swallowing in humans. J Neurophysiol. 2001;85(2):938-50. [PMID: 11160524$]$

18. Zald DH, Pardo JV. The functional neuroanatomy of voluntary swallowing. Ann Neurol. 1999;46(3):281-86. [PMID: 10482257]

19. Smithard DG, O’Neill PA, Martin DF, England RE. Aspiration following stroke: is it related to the side of the stroke? Clin Rehabil. 1997;11(1):73-76. [PMID: 9065363]

20. Heilman KM, Valenstein E. Clinical neuropsychology. 3rd ed. New York (NY): Oxford University Press; 1993.

21. Barer DH. The natural history and functional consequences of dysphagia after hemispheric stroke. J Neurol Neurosurg Psychiatry. 1989;52(2):236-41. [PMID: 2564884]

22. Logemann JA. Evaluation and treatment of swallowing disorders. 2nd ed. Austin (TX): PRO-ED Inc; 1997.

23. Daniels SK, McAdam CP, Brailey K, Foundas AL. Clinical assessment of swallowing and prediction of dysphagia severity. Am J Speech Lang Pathol. 1997;6(4):17-24.
24. Daniels SK, Ballo LA, Mahoney MC, Foundas AL. Clinical predictors of dysphagia and aspiration risk: outcome measures in acute stroke patients. Arch Phys Med Rehabil. 2000;81(8):1030-33. [PMID: 10943750]

25. Horner J, Brazer SR, Massey EW. Aspiration in bilateral stroke patients: a validation study. Neurology. 1993;43(2): 430-33. [PMID: 8437716]

26. Horner J, Massey EW, Riski JE, Lathrop DL, Chase KN. Aspiration following stroke: clinical correlates and outcome. Neurology. 1988;38(9):1359-62. [PMID: 3412582]

27. McCullough GH, Wertz RT, Rosenbek JC. Sensitivity and specificity of clinical/bedside examination signs for detecting aspiration in adults subsequent to stroke. J Commun Disord. 2001;34(1-2):55-72. [PMID: 11322570]

28. Logemann JA, Veis S, Colangelo L. A screening procedure for oropharyngeal dysphagia. Dysphagia. 1999;14(1):44-51. [PMID: 9828274]

29. Rosenbek JC, Robbins JA, Roecker EB, Coyle JL, Wood JL. A penetration-aspiration scale. Dysphagia. 1996;11(2): 93-98. [PMID: 8721066]

30. Goodglass H, Kaplan E. The assessment of aphasia and related disorders. 2nd ed. Philadelphia (PA): Lea \& Febiger; 1984.

31. Miller AJ. The neuroscientific principles of swallowing and dysphagia. San Diego (CA): Singular; 1998.

Submitted for publication December 3, 2004. Accepted in revised form September 26, 2005. 
